\title{
Meckel syndrome
}

INSERM

\section{Source}

INSERM. (1999). Orphanet: an online rare disease and orphan drug data base. Meckel syndrome. ORPHA:564

Meckel syndrome (MKS) is a rare, lethal, genetic, multiple congenital anomaly disorder characterized by the triad of brain malformation (mainly occipital encephalocele), large polycystic kidneys, and polydactyly, as well as associated abnormalities that may include cleft lip/palate, cardiac and genital anomalies, central nervous system (CNS) malformations, liver fibrosis, and bone dysplasia. 